\title{
【Transaction】
}

\section{Emulsifying Properties of $\alpha$-Chitin Nanofibrils Prepared by Aqueous Counter Collision}

\author{
Koichiro Ishida, Shingo Yokota, Tetsuo Kondo\# \\ Graduate School of Bioresource and Bioenvironmental Sciences, \\ Kyushu University, 744, Motooka, Nishi-ku, Fukuoka 819-0395, Japan
}

\begin{abstract}
Renewable and biocompatible nanofibrils derived from natural polysaccharides attract a great attention not only for fiber-reinforced composite materials, but for fibrous emulsifiers for Pickering emulsions (PEs) in the fields of food emulsions, cosmetics, and drug delivery. The PEs are usually stabilized by adsorption of Pickering emulsifiers at oil-water interfaces. This study attempts to examine emulsifying efficiency of chitin nanofibrils that are prepared by aqueous counter collision (ACC) process. Namely, the obtained chitin nanofibrils (ACC-ChNFs) worked to assist formation of PEs from the mixture of water and typical organic solvents as well as an edible canola oil. Their oil-water interfacial tension, droplet diameter distribution and surface potential of oil droplets were compared with the case using ACC-cellulose nanofibrils as an emulsifier. As a result, ACC-ChNFs exhibited superior properties allowing stabilization of oil droplets with various oilwater interfacial tension values. Moreover, emulsification characteristics of canola oil-based emulsions in the system of ACC-ChNFs were quite different from those of the ACC-CNFs. This might be based on the unique interfacial adsorption of cationic ACC-ChNFs onto canola oils involved in complexing with anionic fatty acids.

(Received 7 May, 2021; Accepted 25 June, 2021)
\end{abstract}

\section{Introduction}

Chitin, one of biocompatible and biodegradable polysaccharides, is a promising candidate for Pickering emulsifiers, similarly to cellulose nanofibrils (CNFs). At the molecular scales, chitin is a liner polymer composed of $N$-acetyl-D-glucosamine (GlcNAc) residues connected by $\beta$-glucoside linkages. The $N$-acetyl groups at the $\mathrm{C}-2$ position of GlcNAc residues are partially hydrolyzed, resulting in presence of dissociable amino groups as a minor but significant moiety. Chitin is classified as $\alpha$ - and $\beta$ chitin from the viewpoint of either anti-parallel or parallel molecular packing in their crystalline structure, respectively [1,2]. Among the chitins, the most abundant $\alpha$-chitin having crystalline allomorphs of anti-parallel molecular packing is mainly extracted from shells of crabs [3] and shrimps [4].

The growing willingness to develop bioactive chitin-based nanomaterials is induced from the advantageous fact that chitin nanofibrils (ChNFs) have high specific surface areas [5], high mechanical strength [6], as well as their bioactivities such as bone regeneration [7] and hair growth-promoting [8]. ChNFs are produced by various ways proposed e.g., mechanical defibrillation [9], TEMPO-mediated oxidation [10], sonication treatment in acidic media [11], and the aqueous counter collision (ACC) method $[12,13]$ that is employed in the current study.

According to the previous studies on ACCproduced cellulose nanofibrils (ACC-CNFs), the advantage of ACC method [14, 15], which is also capable to prepare chitin nanofibrils $[12,13]$ and collagen nanofibrils $[16,17]$ solely by impinging of a pair of water jets containing bio-based raw materials, is considered to provide characteristic nano-objects exhibiting unique amphiphilic properties in water [18] that would be helpful to stabilize oil-droplets as a Pickering emulsion (PE) by the oil-surface coverage $[19,20]$.

The PE [21,22] is, in general, a kind of the emulsion stabilized by solid particles such as clays [23], polymer latexes [24], bacteria [25], and fibers [26]. In recent years, natural polymer-based objects attract

\# corresponding author: Tetsuo Kondo (E-mail: tekondo@agr.kyushu-u.ac.jp) 
great interests as Pickering emulsifiers and stabilizers in the fields of food colloids, cosmetics, painting, and drug delivery due to their biocompatibility and bioactivities [27, 28].

Understanding of basic characterizations and thereby applications of PEs stabilized by ChNFs are, however, limited [29], when compared with massive information on cellulose nanofibrils (CNFs)-assisted PEs. Typically listed, Larbi et al. reported that the ChNFs-stabilized PE exhibited higher stability than the effect due to chitin nanocrystals (ChNCs) having a low aspect ratio [9]. Huang et al. reported that the ChNFs-stabilized PE exhibited antibacterial activities [30]. Composite particles with ChNFs [31] or ChNCs [32] were reported to be prepared using Pickering emulsion polymerization techniques. It is noted that our previous paper [12] showed unique physical properties different from that of ACC-CNFs [12].

In the current study, the attempt has been made, therefore, to achieve emulsification using of $\alpha$-chitin nanofibrils prepared by ACC (ACC-ChNFs), and then some distinctive effects of ACC-ChNFs were found to work as an assistant for formation of their Pickering emulsification. The PEs obtained with ACC-ChNFs exhibit a superior stability and versatility to the bacterial cellulose-based ACC-CNFs, although the characteristics of PEs were sensitive to the nanofiber concentration and $\mathrm{pH}$ condition.

\section{Experimental}

\subsection{Materials}

$\alpha$-Chitin powder and hydrochloric acid were purchased from FUJIFILM Wako Pure Chemical Corporation (Osaka, Japan). Cyclohexane (>99.5\%), dichloromethane (DCM; $>99.0 \%)$, toluene (>99.5\%), sodium hydroxide $(>96.0 \%)$, and Calcofluor White (CFW) were purchased from Sigma-Aldrich Japan, LLC., Tokyo, Japan. Vinyl acetate (VAc; $>99.0 \%$ ) was purchased from Tokyo Chemical Industry Co., Ltd., Tokyo, Japan. Canola oil was purchased from The Nisshin OilliO Group, Ltd., Tokyo, Japan. All reagents were used without further purification. Ultrapure water produced with a NANO pure Diamond (Thermo Fisher Scientific, Inc., Waltham, United States) was used for interfacial tension measurements. In other experiments, deionized water was used.

\subsection{Preparation of polysaccharide-based nanofibrils}

\subsubsection{ACC-ChNFs}

The aqueous dispersion of ACC-ChNFs was prepared according to our previous report [12]. Namely, $\alpha$-chitin powder was suspended in deionized water for the nano-pulverization by an ACC system (CNNT, Incheon, Korea) with $200 \mathrm{MPa}$ of ejecting pressure and 60 Pass of the repeated cycle, respectively. The obtained aqueous dispersion containing ACC-ChNFs was diluted to the prescribed concentration with deionized water.

\subsubsection{ACC-CNFs}

Bacterial cellulose (BC) pellicles, which is composed only of cellulose component and thus "pure cellulose", were used for preparation of ACC-CNFs as a reference in this ChNFs study. BC pellicles obtained according to our previous report [33] was defibrillated with food mixer for $90 \mathrm{~s}$ prior to the ACC treatment with the same condition as for ACC-CNFs at $200 \mathrm{MPa}$ and 60 Pass [20,33]. The obtained aqueous dispersion of ACC-CNFs was also diluted to be prescribed concentration with deionized water.

\subsection{Characterization of polysaccharide-based nanofibrils}

\subsubsection{Atomic force microscopy}

Morphological observation of ACC-ChNFs and ACC-CNFs was carried out using an atomic force microscope (Asylum MFP-3D Origin; Oxford Instruments, Abingdon-on-Thames, United Kingdom) equipped with a silicon cantilever (OMCL-AC160TS-R 3; Olympus Corp., Tokyo, Japan) in the AC mode under ambient conditions. Aqueous dispersions containing $1.0 \times 10^{-3} \mathrm{wt} \%$ nanofibrils were dropped on a glass slide substrate, then dried at $50{ }^{\circ} \mathrm{C}$ for $\mathrm{AFM}$ observation. Length and height values of ACC-ChNF were estimated from the obtained AFM height images.

The aspect ratios of each nanofiber were calculated using the following formulae:

$$
\text { Aspect ratio }=2 l /(h+w)
$$

where, $l$ is length of nanofibrils, $h$ is height of nanofibrils, and $w$ is width of nanofibrils, respectively. The specific surface areas (SSA) of each nanofibril were estimated assuming square pole with no branches using the following equation:

$$
\mathrm{SSA}=2(l h+l w+h w) / \rho l h w
$$

where, $\rho$ is the density of $\alpha$-chitin crystal; $1.46 \mathrm{~g} \mathrm{~cm}^{-3}$ [34] and cellulose I crystal; $1.62 \mathrm{~g} \mathrm{~cm}^{-3}$ [35].

\subsubsection{Determination of degree of deacetylation (DDA)}

The DDA of $\alpha$-chitin was determined from the $\mathrm{C} / \mathrm{N}$ atom ratio according to the previous elemental analysis method [36]. 


\subsubsection{Zeta potential measurements}

Zeta potential measurements of aqueous dispersions of $0.01 \%(\mathrm{w} / \mathrm{w})$ ACC-ChNFs and ACCCNFs were performed using a Malvern Zetasizer Nano ZS (Malvern Instruments Ltd., Worcestershire, United Kingdom) with a disposable cells (DTS 1070, Malvern Instruments) at $25{ }^{\circ} \mathrm{C}$. The $\mathrm{pH}$ values were adjusted from the initial value of 5.5 of both the dispersions to the prescribed values with diluted $\mathrm{HCl}$ aqueous solution and diluted $\mathrm{NaOH}$ aqueous solution prior to measurements. The formula of Smoluchowski [37] was used to calculate zeta potential values, which were averaged from 15 times measurements.

\subsection{Preparation of PEs stabilized by polysaccharide- based nanofibrils}

To investigate interfacial adsorption of polysaccharide-based nanofibers, 4 types of typical organic solvents with different interfacial tension values between oil and water were used in addition to canola oil. $5 \mathrm{~mL}$ of aqueous dispersions containing nanofibrils and $5 \mathrm{~mL}$ of oils (cyclohexane, toluene, canola oil, DCM, or VAc) were poured into a $20 \mathrm{~mL}$ glass vessel to be mixed for emulsification with a vortex mixer (Vortex-Genie 2; KENIS LIMITED, Osaka, Japan) for $2 \mathrm{~min}$ at room temperature.

\subsection{Characterization of PEs}

\subsubsection{O/W Interfacial tension measurements}

Interfacial tension values between each oil and ultrapure water were measured by pendant drop shape analysis using a Drop Master DM300 (Kyowa Interface Science CO., Ltd., Saitama, Japan): As the shape of the drop hanging from a needle is determined from the balance of forces which include the surface tension of the liquid being investigated, the surface or interfacial tension can be related to the drop shape by the equation;

$$
\gamma=-\Delta \rho \mathrm{gR}_{0}{ }^{2} / \beta
$$

where $\gamma$ is the surface tension, $\Delta \rho$ is the density difference between fluids, $\mathrm{g}$ is the gravitational constant, $R_{0}$ is the drop radius of curvature at the apex and $\beta$ is the shape factor. Measurements were conducted at least 10 times, and the average values were determined as the interfacial tension value.

\subsubsection{Field emission scanning electron microscopy (FE-SEM)}

PEs prepared from cyclohexane and aqueous dispersions containing $0.1 \mathrm{wt} \%$ of polysaccharidebased nanofibrils were diluted three times with excess deionized water. Some of the droplets were dropped on a carbon tape to be lyophilized. Then, osmium tetroxide coating was performed as a pretreatment using an HPC-1 SW system (Vacuum Device Corp., Ibaraki, Japan) for FE-SEM observation. The FE-SEM of an SU 8000 scanning electron microscope (Hitachi High-Tech Corp., Tokyo, Japan) was employed at an acceleration voltage of $2.0 \mathrm{kV}$.

\subsubsection{Confocal laser scanning microscopic observation of PEs}

To remove non-adsorbed nanofibrils from an aqueous phase, $10 \mathrm{~mL}$ of the $\mathrm{O} / \mathrm{W}$ mixture was washed with $50 \mathrm{~mL}$ of deionized water 3 times using a separation funnel. The washed samples were further mixed with $15 \mathrm{~mL}$ of $1.3 \times 10^{-3} \mathrm{wt} \%$ calcofluor white (CFW) aqueous solution for fixing a fluorescent probe of chitin and cellulose, then shaken at $300 \mathrm{rpm}$ for 10 min in a $50 \mathrm{~mL}$ glass vessel under shade conditions. The fluorescence-stained samples were purified with $100 \mathrm{~mL}$ of deionized water 3 times using a separation funnel to remove the non-adsorbed $\mathrm{CFW}$. The samples thus purified were transferred to a glassbottom dish for observation by confocal scanning microscopy. Presence of polysaccharide-based nanofibrils adsorbed at the oil-water interface were examined using a Leica SP8 (Leica microsystems, Wetzlar, Germany) equipped with a photomultiplier tube detector (Leica microsystems). The wavelength of the excitation and the detection of the fluorescent emission were set as $405 \mathrm{~nm}$ and 415-475 nm, respectively.

\subsubsection{Droplet diameter measurements}

Droplet diameters of the PEs were measured and averaged using a laser diffraction particle size analyzer (SALD-200 V, Shimadzu Corporation, Kyoto, Japan) equipped with a quartz cell. A portion of droplets were diluted with excess deionized water prior to measurements.

\subsubsection{Zeta potential measurements of canola oil emulsion system}

To investigate interfacial characteristics of oil droplets suspended in emulsions, their zeta potentials depending on the concentration of ACC-ChNFs were measured using a Malvern Zetasizer Nano ZS (Malvern Instruments Ltd.) with disposable cells (DTS 1070, Malvern Instruments) at $25{ }^{\circ} \mathrm{C}$. Prior to measurements, $1 \mathrm{~mL}$ of freshly prepared emulsified phase composed of oil droplets stabilized by the ACCChNFs was washed with deionized water 3 times to remove non-adsorbed ACC-ChNFs in an aqueous phase. Eventually, the washed droplets were suspended in $15 \mathrm{~mL}$ of deionized water. The obtained 
suspensions were hand-shaken and transferred into a measurement cell to prevent aggregation of droplets. Average values of the zeta potential of each sample was calculated based on at least 5 times measurements.

\section{Results and discussion}

\subsection{Characteristics of polysaccharide - based nanofibrils}

Fig. 1 shows AFM topographical images of both ACC-ChNFs and ACC-CNFs. The values of sizes, aspect ratio and specific surface areas (SSA) for the ACC-ChNFs and ACC-CNFs were summarized in Table 1. The degree of deacetylation (DDA) values of chitin raw material were determined as $1.3 \%$ based on

(a)

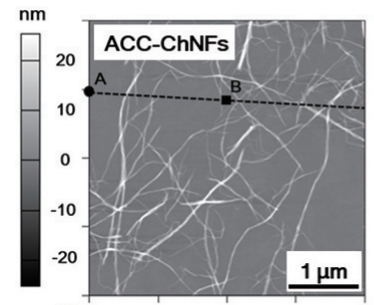

(b)
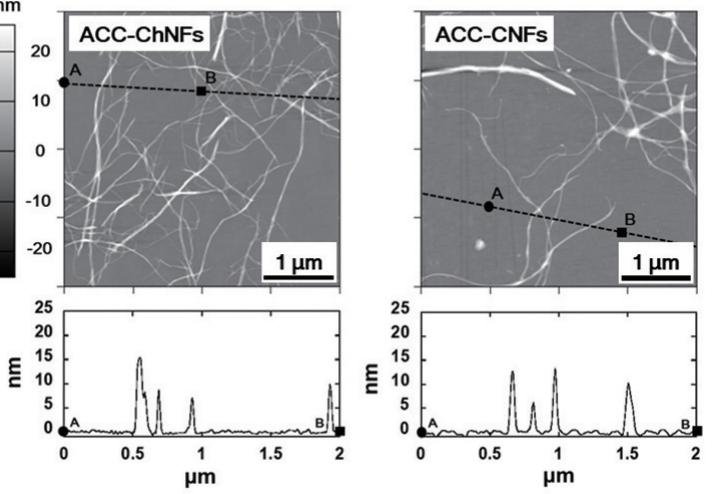

Fig. 1 (a) AFM topographical images of ACC-ChNFs and ACC-CNFs. (b) Cross section profiles along the between $\mathrm{A}$ and $\mathrm{B}$ dots on the line in each AFM image.

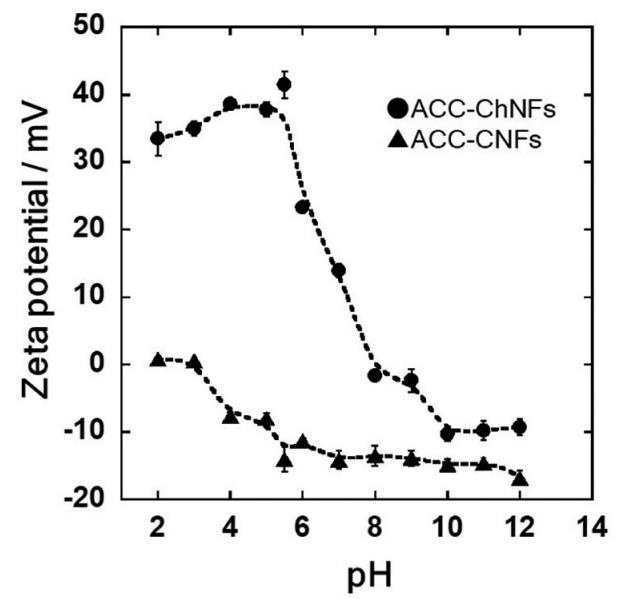

Fig. 2 pH-Dependence of zeta potential values for each polysaccharide-based nanofiber. the elemental analysis.

Fig. 2 draws that the zeta potential values of ACC-ChNFs and ACC-CNFs depending on $\mathrm{pH}$ values of their aqueous dispersion media. ACC-ChNFs exhibited positive zeta potential values $(30-40 \mathrm{mV})$ under acidic conditions ( $\mathrm{pH}$ 2-5.5). With increase in $\mathrm{pH}$ values in the range of $6-10$, the absolute values of zeta potentials were then significantly decreased. Eventually, ACC-ChNFs exhibited negative zeta potentials under alkali conditions $(\mathrm{pH}>10)$. The behavior is presumably due to a balance of association and dissociation of amino and/or hydroxy groups on the surface of ACC-ChNFs. Namely, their surface exhibits a pH-responsive characteristic. In contrast, negative zeta potential values of ACC-CNFs appeared all through from acidic, neutral till alkali pH conditions, although it was not a drastic behavior, different from ACC-ChNFs. This implies that hydroxy groups on the surface of ACC-CNFs were predominant moieties that do not exhibit their pH-responsive surface potentials.

\subsection{Emulsifying properties of polysaccharide-based nanofibrils to typical organic solvents}

To characterize emulsifying abilities of polysaccharide-based nanofibrils, agitated mixtures containing various oils and water dispersions of either ACC-ChNFs or ACC-CNFs were investigated. Fig. 3 plots the relative ratio of emulsified volumes after 5 min of mixing aqueous dispersions containing $0.02 \mathrm{wt} \%$ nanofibrils with oils having different oil-water interfacial tensions (Table 2). Negligible emulsifications using VAc as an oil phase $(\times$ in Fig. 3$)$ were observed in both nanofibril systems. In the case of the ACC-CNFs (b in Fig. 3), the emulsified volume was in a sense sensitive to interfacial tension of oils. On the other hand, the ACC-ChNFs system (a in Fig. 3) allowed the mixture to be emulsified more favorably than the ACC-CNFs one, possibly due to a remarkable difference of surface characteristics between ACCChNFs and ACC-CNFs. The surface of ACC-ChNFs owns positively charged amino groups, whereas the surface charge of ACC-CNFs would be negligible due to lack of dissociable moieties. Jia et al. reported that emulsifying properties decreased with an increase in the surface charge on the TEMPO-oxidized CNF

Table 1 Length, height, width, aspect ratio, and SSA values of ACC-ChNFs and ACC-CNFs

\begin{tabular}{cccccc}
\hline & Length $/ \mu \mathrm{m}$ & Height $/ \mathrm{nm}$ & Width $/ \mathrm{nm}$ & Aspect ratio & $\mathrm{SSA} / \mathrm{m}^{2} \mathrm{~g}^{-1}$ \\
\hline ACC-ChNFs & $2.3 \pm 1.0$ & $6.1 \pm 3.1$ & $10 \pm 6[12]$ & 286 & 362 \\
ACC-CNFs & $4.4 \pm 2.3[38]$ & $10.1 \pm 4.2[20]$ & $22 \pm 13[12]$ & 274 & 179 \\
\hline
\end{tabular}


Table 2 Interfacial tension values between various oils and water.

\begin{tabular}{cccccc}
\hline & Cyclohexane & Toluene & Canola oil & DCM & VAc \\
\hline $\begin{array}{c}\text { lnteriacial } \\
\text { tension } / \mathrm{mNm}^{-1}\end{array}$ & $51.1 \pm 1.0$ & $31.5 \pm 0.4$ & $28.0 \pm 0.4$ & $27.9 \pm 0.6$ & $13.5 \pm 0.6$ \\
\hline
\end{tabular}
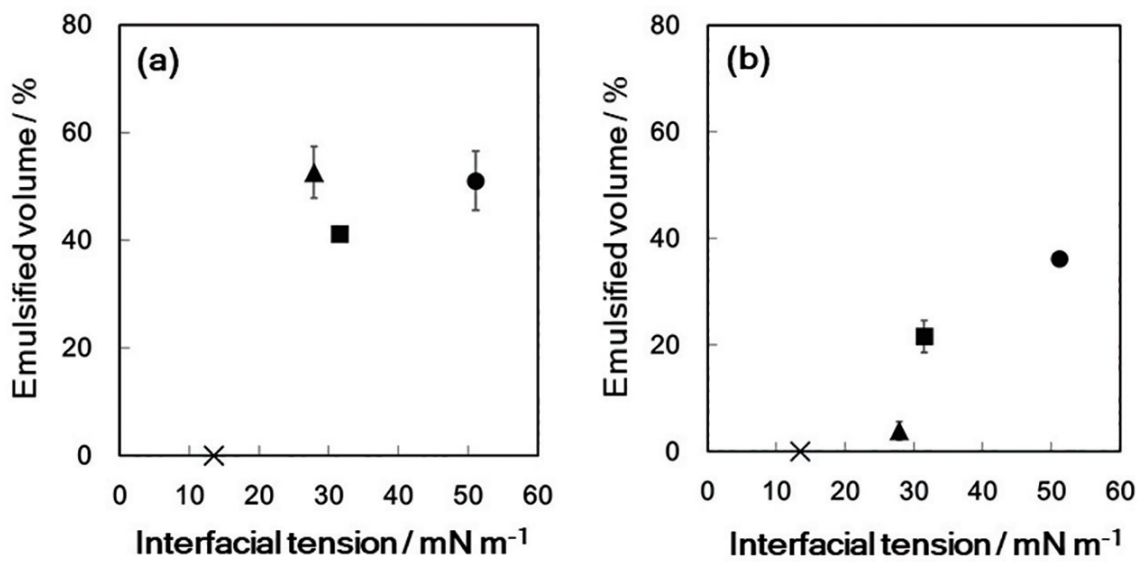

Fig. 3 Emulsified volumes of PEs prepared from various oils and aqueous dispersions containing 0.02 wt\% of (a) ACC-ChNFs and (b) ACC-CNFs. Circle (@); cyclohexane, square ( $\square$ ); toluene, triangle $(\mathbf{\Delta})$; DCM, and cross $(\times)$; VAc.

(TOCNF) [39]. Wu et al. reported that PEs were highly stabilized using protein nanoparticles due to inhibition of the coalescence of oil droplets by the electrostatic repulsive force between the Pickering emulsifiers [40]. Taking them into accounts, since ACC-ChNFs in the current study exhibited $41.5 \pm 2.0 \mathrm{mV}$ as a zeta potential value to be positively charged, the nanofibers presumably behaved as an electrostatic stabilizer for the PEs.

Moreover, emulsification occurred in the extremely low concentrations $(=0.02 \mathrm{wt} \%)$ for both of the nanofibrils, indicating that the polysaccharidebased nanofibrils prepared by ACC adsorb preferably to the oil surface [19] and thereby stabilize oil-water interfaces, according to the lyophilized PE shell structures constructed from nanofibrils as shown in Fig. 4. It is noted that all dispersed phases were quickly dispersed into water, indicating that the obtained emulsions were in oil-in-water $(\mathrm{O} / \mathrm{W})$ type as

\section{ACC-ChNFs}

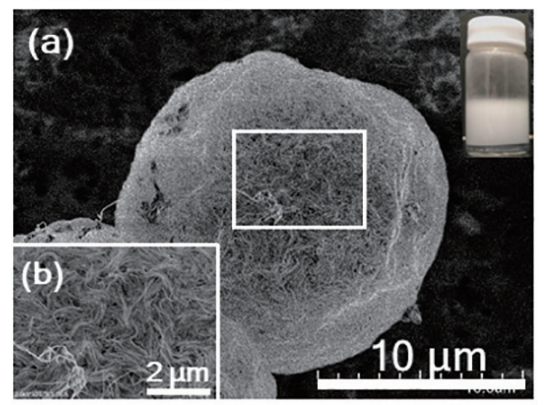

for the same case for that for wood-based ACC-CNFs [19]. In this sense, the both nanofibrils are supposed to act as a Pickering emulsifier.

Fig. 5 shows concentration dependency of emulsified volumes of the PEs 5 min after standing still. The emulsified volume in the PEs stabilized by ACC-ChNFs exhibited clearly higher than that in PEs stabilized by ACC-CNFs except the case of the canola oil (this will be discussed later). Namely, the ACCChNFs emulsified favorably oil droplets irrespective of the oil type, independent of their interfacial tensions (Fig. 5ab). As for ACC-CNFs system (Fig. 5b), PEs of DCM were negligibly formed in the ACCCNFs system all through the entire concentration range measured, even if the concentration increased. In other words, ACC-ChNFs induced the emulsification of DCM, differently from ACC-CNFs. In addition, the concentration-dependency in changes of emulsified volume in the ACC-ChNFs system are

\section{ACC-CNFs}

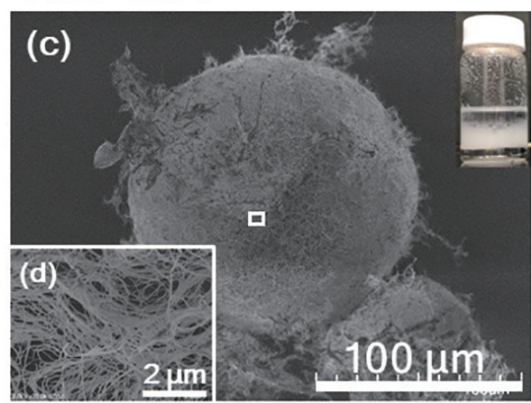

Fig. 4 FE-SEM (a, c) low magnified images of lyophilized PEs, and (b, d) zoomed images of the enclosed regions. 

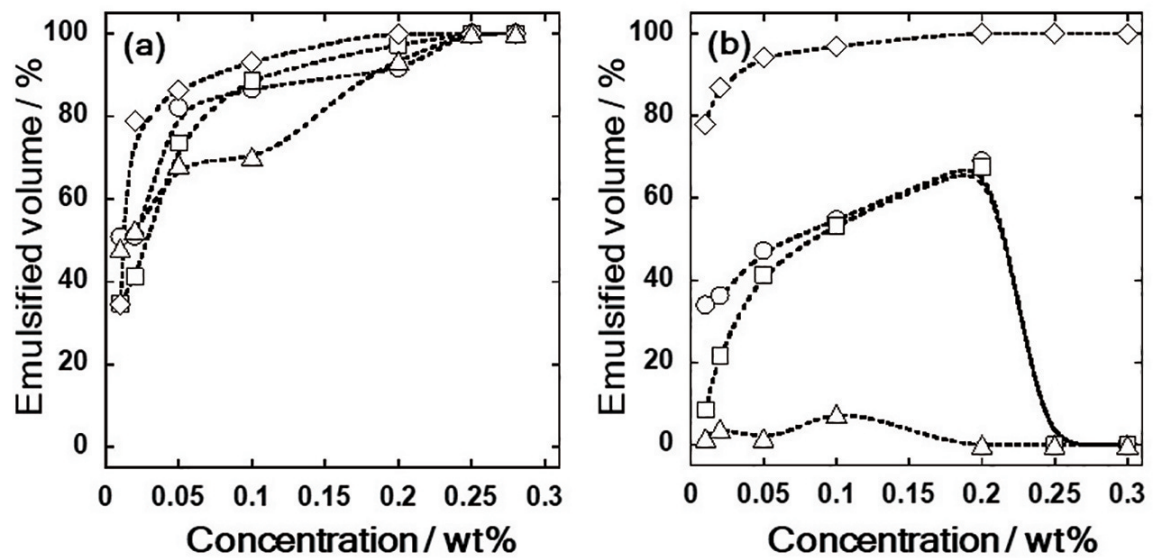

Fig. 5 Emulsified volumes of the PE stabilized by (a) ACC-ChNFs and (b) ACC-CNFs. Circle (O): cyclohexane, square $(\square)$ : toluene, diamond $(\diamond)$ : canola oil, and triangle $(\triangle)$ : DCM.

quite different from those of the ACC-CNFs system.

In general, stability of nanofiber networks become stronger with an increase in the nanofiber concentration due to increase in their crosslinking points. Goi et al. reported that aqueous dispersion of TOCNF exhibited two significant roles as an emulsifier and a dispersion stabilizer [41]. In the current study, emulsified volumes of PEs were increased with increasing in concentration of ACCCNFs up to $0.20 \mathrm{wt} \%$, which might be due to the increase in the total surface area of the individual PE oil droplets possibly for adsorption of the nanofibers. Then, the emulsified volume was rapidly decreased over $0.25 \mathrm{wt} \%$ in the cases of cyclohexane and toluene (Fig. 5b). This indicates that the higher concentration prevents the adsorption of ACC-CNFs onto oil droplets because of generation of stable nanofiber networks. Tanaka et al. reported that viscosity of TOCNF dispersion significantly increased until the TOCNF concentration reaches the critical concentration because of the increase in crosslinking points [42]. This implies that the stirring energy of the vortex mixing did not reach a threshold for the collapse of nanofiber networks in the dispersion of ACC-CNFs. As a result, the surface coverage for the oil droplet due to adsorption of ACC-CNFs was possibly inhibited at a high concentration of their aqueous dispersion.

In contrast, the ACC-ChNFs did not exhibit such a maximum point in emulsified volumes of PEs. Although the aspect ratio between the two nanofibers was similar (Table 1), the length value of ACC-ChNFs was approximately half of ACC-CNFs length and ACC-ChNFs was also thinner than ACC-CNFs. Furthermore, the electrostatic repulsion force between ACC-ChNFs is supposed to be higher than that of ACC-CNFs by taking account of the zeta potential values (Fig. 2). Therefore, the mobility of the ACC-ChNFs in an aqueous medium is likely to be higher than that of ACC-CNFs. This assumption refers to the idea that ACC-ChNFs could adsorb more favorably onto the oil-water interface to exhibit superior emulsifying effects even under the higher nanofiber concentration. Investigations are still required to discuss quantitative relationships between emulsification properties and mixing energy, mobility and/or adsorbability of the nanofibrils.

\subsection{Emulsifying properties of polysaccharide-based nanofibrils to canola oil}

Investigation of the emulsion with edible natural oils and emulsifier/stabilizers is worth considering as a fundamental study for food, pharmaceutic and medical applications. Therefore, PEs of a canola oil with ACC-ChNFs were also investigated in comparison of adding ACC-CNFs.

In the case of the canola oil, $\mathrm{O} / \mathrm{W}$ emulsion for both nanofibrils that were prepared by ACC process were obtained, and the volume of emulsified phase was larger than other organic solvents, as shown in Fig. 5ab. In particular, although the oil-water interfacial tension of the canola oil was similar to that of DCM (Table 2), ACC-ChNFs induced the emulsification of DCM, whereas ACC-CNFs exhibited negligible emulsifying effects.

To observe the adsorption of the two nanofibrils on the surface of canola oil droplets in the PEs, confocal laser scanning microscopy was employed because it was hard to perform freeze-drying of the canola oil droplets. Fig. 6 shows the fluorescent images of diluted emulsion phases stained with $\mathrm{CFW}$ as a probe for the localization of polysaccharides. This indicated that the interfacial stabilization appeared to 


\section{ACC-ChNFs}
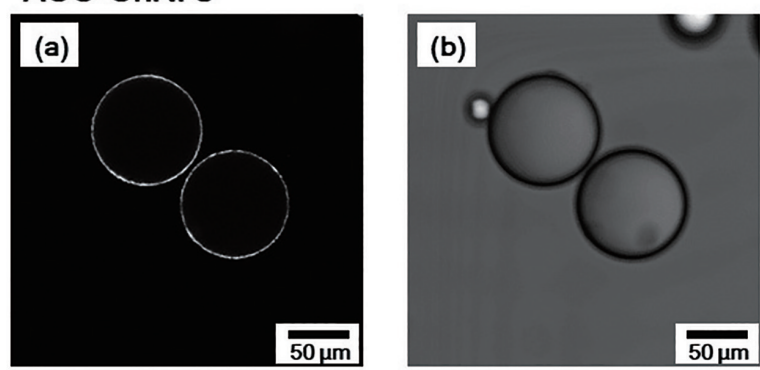

\section{ACC-CNFs}
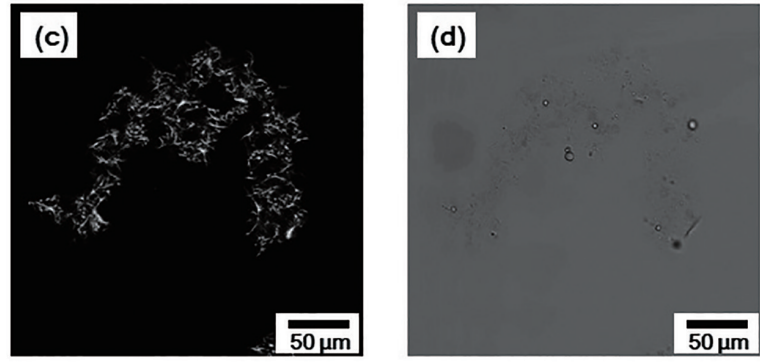

Fig. 6 Confocal laser scanning microscopic sectioning images of PEs prepared from canola oil and aqueous dispersions at $0.25 \mathrm{wt} \%$ of the two nanofibers. (a, c) Fluorescent images and (b, d) bright field images.

be achieved by surface coverage of ACC-ChNFs at the interface on the canola oil droplets, where the fluorescent emission of the CFW attaching to the nanofibrils were clearly observed (Fig. $6 \mathrm{ab}$ ). On the other hand, it appeared that the small droplets were trapped and stabilized by network of ACC-CNFs (Fig. $6 c d)$.

A canola oil is a complex oil that contains several fatty acids such as oleic acid, linoleic acid, $\alpha$-linoleic acid. Previously, the emulsification phenomenon in the system of water and fatty acids without any other additives was reported [43], which might be caused by surfactant-like chemical structures of fatty acids having hydrophobic long alkyl chains and hydrophilic carboxylic acids. In fact, the canola oil provided the emulsion in the nanofiber-free system (dotted lines in Fig. $7 \mathrm{a}-\mathrm{c}$ ), and it formed relatively smaller droplets having ca. $8 \mu \mathrm{m}$ in volume average droplet diameter $\left(D_{V}\right)$ than those of ca. $60-121 \mu \mathrm{m}$ in $D_{V}$ obtained in the ACC-ChNFs system. This indicates that the emulsification mechanism for the canola oil is different in the systems between ACC-ChNFs and ACC-CNFs. According to Fig. 6ac, the predominant effect of ACCCNFs may be a dispersion stabilization of canola oil droplets, whereas ACC-ChNFs were allowed to cover the surface oil droplets, as well as the self-stabilization of fatty acids in the canola oil. It should be added that the emulsified phase of the canola oil emulsion prepared in the nanofiber free system are collapsed within 8 days, and the emulsified phase of canola oil/ $0.02-0.30$ wt $\%$ polysaccharide-based nanofibril systems stayed at least for 4 months.

Fig. 7 shows the $D_{V}$ and number average droplet diameter $\left(D_{N}\right)$ of nanofibril-stabilized canola oil droplets for both cases of ACC-ChNFs and ACCCNFs. It is noted that the canola oil droplets stabilized by 0.20 wt $\%$ of ACC-ChNFs suspension exhibited relatively narrow $D_{V}$ distribution. This indicates that the canola oil droplets stabilized by ACC-ChNFs were established, soon after formation of the canola oil/ water emulsion.

The adsorption of anionic surfactants onto the chitin surface with cationic amino groups due to the electrostatic attraction was previously reported $[44,45]$. Therefore, the zeta potential values of the canola oil/ACC-ChNFs emulsion system in the current study were measured as shown in Fig. 8. There is no significant change in zeta potential values (ca. $-21 \mathrm{mV}$ ) when the ACC-ChNF concentration is in the range of $0.20-0.28 \mathrm{wt} \%$. The $\mathrm{pH}$ values of aqueous dispersion containing droplets at $0.20 \mathrm{wt} \%$ of ACCChNFs was kept as 6.8, until the negative zeta potential turned to positive value of $(+9.5 \pm 3.5 \mathrm{mV})$ at $\mathrm{pH}$ of 4.0. Therefore, the negative zeta potential values might be due to the slight dissociation of positive amino groups of the PE shells constructed from ACC-ChNFs under a neutralized condition by negative carboxylic acids of the canola oil. The absolute values of negative zeta potential of canola oil droplets increased with decreasing in the concentration of ACC-ChNFs, and eventually the zeta potential value in the nanofiber-free condition reached ca. -51 mV. Notably, the concentration-dependent changes of zeta potential values corresponded to concentration-dependent droplet size distribution as shown in Fig. 7, indicating that presence of the critical concentration for PEs stabilized only by ACC-ChNFs.

\section{Conclusion}

Polysaccharide-based nanofibrils, ACC-ChNF, as well as ACC-CNFs, which were prepared by ACC, were found to stabilize oil-water interface as Pickering emulsifiers. Interfacial adsorption of ACCCNFs was likely to correlate to the specific oil-water interfacial tension values, whereas the ACC-ChNFs exhibited more efficient emulsifier performance in the wide range of oil-water interfacial tension value. 

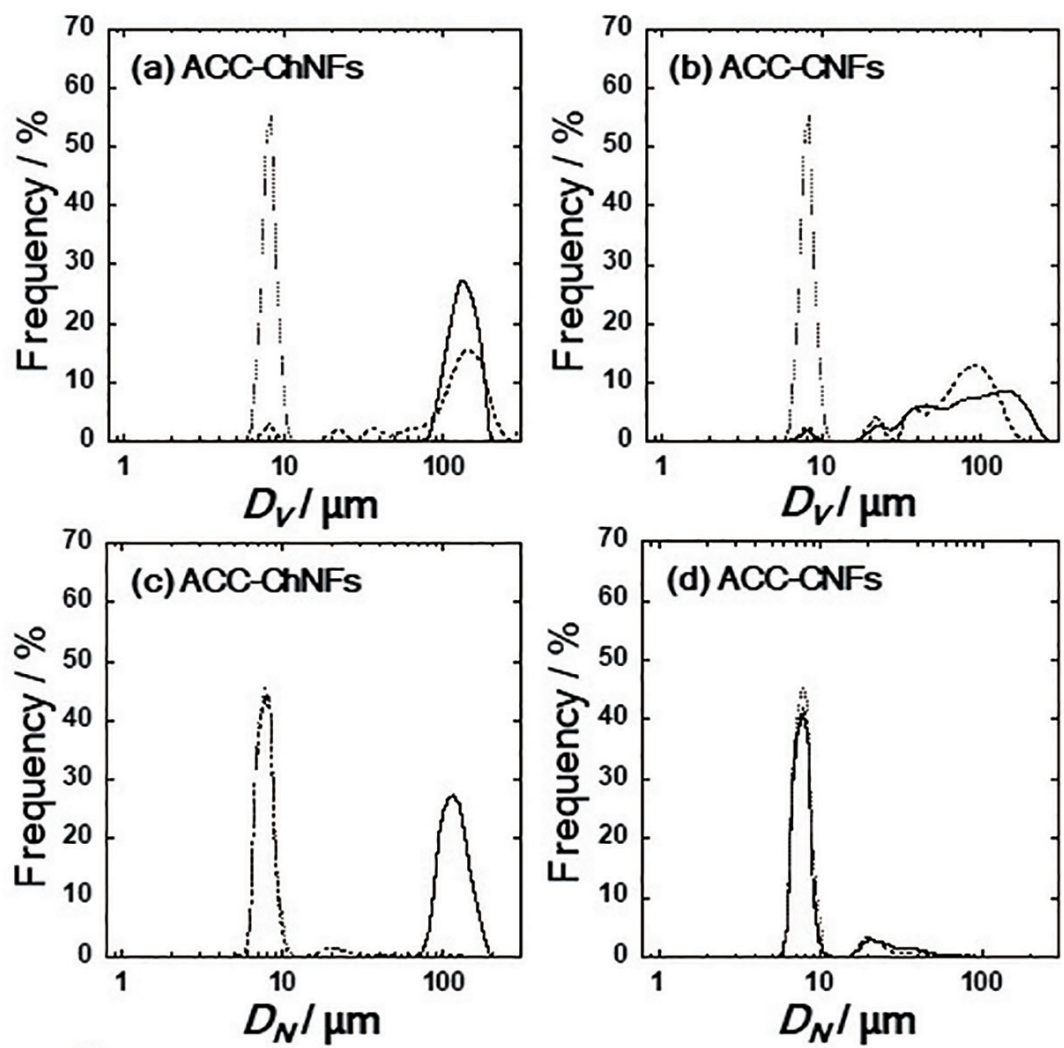

(e)

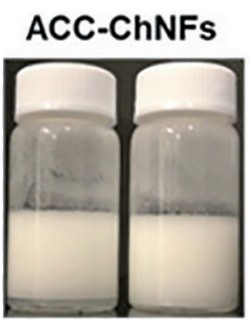

ACC-CNFs
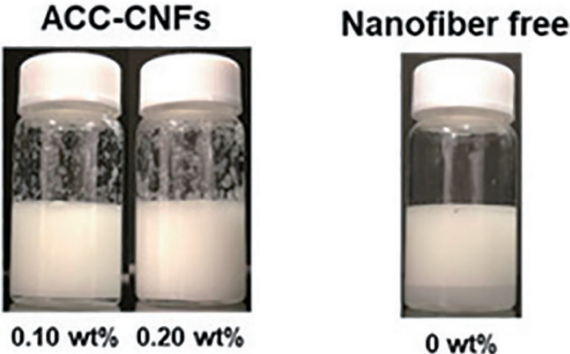

Fig. 7 Droplet diameter distributions of canola oil droplet assisted by ACC-ChNFs, ACC-CNFs, and canola oil itself (nanofiber free condition). (a, b) volume average diameter, (c, d) number average diameter. Concentration of nanofibers were $0.20 \mathrm{wt} \%$ (solid line), $0.10 \mathrm{wt} \%$ (dashed line), and $0 \mathrm{wt} \%$ (dotted line), respectively. (e) Photo images of canola oil emulsion stabilized by polysaccharide-based nanofibers.

Irrespective of the aqueous suspension concentration, ACC-ChNFs were allowed to stabilize PEs in the aqueous dispersion phase, which may be owing to their higher electrostatic repulsion force and mobility. Among the combination of oil/aqueous suspension of nanofibrils in PEs studied, the stability of emulsions for canola oil were the highest, and it was remarkably improved by adding the polysaccharidebased nanofibrils to the canola oil/water alone.

Concerning canola oil emulsions stabilized by the two polysaccharide - based nanofibrils, more complicated behaviors were monitored, which may be attributed to the emulsification mechanism due to not only characteristics of nanofibrils, but canola oil itself. It is noted that the emulsified volumes and surface charge of oil droplets were sensitive to the

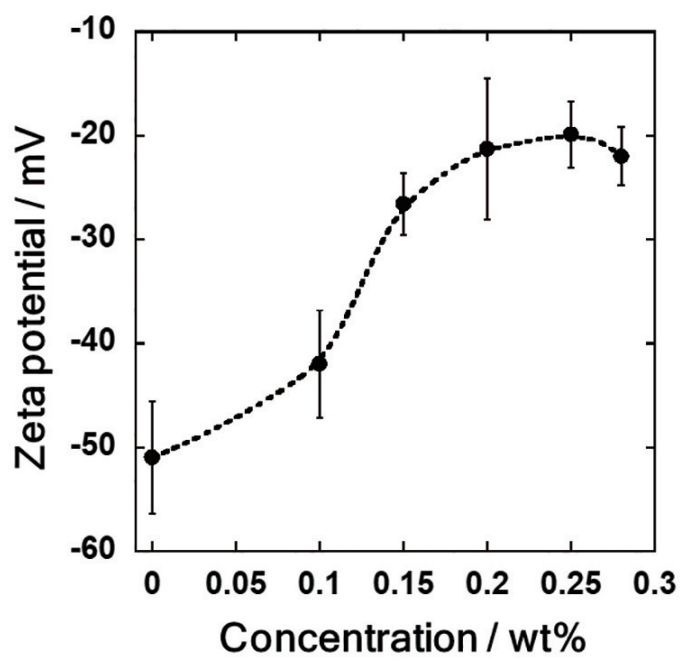

Fig. 8 Concentration-dependence of zeta potential values for canola oil droplets. 
concentration of ACC-ChNFs, differently from the case for ACC-CNFs. When their concentration was set to be higher than $0.20 \mathrm{wt} \%$, interfacial adsorption of ACC-ChNFs was most likely to allow the oil droplets to be stabilized, rather than self-stabilization effects of the canola oil.

Accordingly, this study has highlighted the characteristics in the emulsification behaviors using ACC-ChNFs as an emulsifier for high versatility and thereby for the functional design of PEs in the field of food emulsion, cosmetics, and drug delivery using bioactive chitin objects.

\section{Acknowledgement}

The authors thank for Prof. Noriyuki Igura from Kyushu University for his help in particle size analyze of emulsion. We also thank for The Service Centre of the Elementary Analysis of Organic Compounds, Faculty of Science at Kyushu University for elemental analysis. This work was supported by JSPS KAKENHI Grant Numbers JP18K05767 and by Kyushu University Platform of Inter - / Transdisciplinary Energy Research (Q-PIT).

\section{References}

1. D. Carlstrom, J. Biophys. Biochem. Cytol., 3, 669 (1957).

2. N. E. Dweltz, Biochim. Biophys. Acta, 51, 283 (1961).

3. P. Y. Chen, A. Y. M. Lin, J. McKittrick and M. A. Meyers, Acta Biomater., 4, 587 (2008).

4. D. Raabe, P. Romano, C. Sachs, H. Fabritius, A. AlSawalmih, S. Yi, G. Servos and H. G. Hartwig, Mater. Sci. Eng. A., 421, 143 (2006).

5. R. N. Gao, Y. Lu, S. L. Xiao and J. Li, Sci. Rep., 7, 4303 (2017).

6. Y. Bamba, Y, Ogawa, T. Saito, L. A. Berglund and A. Isogai, Biomacromolecules, 18, 4405 (2017).

7. M. Kawata, K. Azuma, H. Izawa, M. Morimoto, H. Saimoto and S. Ifuku, Carbohydr. Polym., 136, 964 (2016).

8. K. Azuma, R. Koizumi, H. Izawa, M. Morimoto, H. Saimoto, T. Osaki, N. Ito, M. Yamashita, T. Tsuka, T. Imagawa, Y. Okamoto, T. Inoue and S. Ifuku, Int. J. Biol. Macromol., 126, 11 (2019).

9. F. Larbi, A. Garcia, L. J. del Valle, A. Hamou, J. Puiggali, N. Belgacem and J. Bras, Carbohydr. Polym., 196, 385 (2018).

10. Y. M. Fan, H. Fukuzumi, T. Saito and A. Isogai, Int
J. Biol. Macromol., 50, 69 (2012).

11. Y. M. Fan, T. Saito and A. Isogai, Biomacromolecules, 9, 1919 (2008).

12. R. Kose and T. Kondo, Sen'i Gakkaishi, 67, 91 (2011).

13. W. B. Ye, S. Yokota, Y. M. Fan and T. Kondo, Cellulose, 28, 2167 (2021).

14. T. Kondo, M. Morita, K. Hayakawa and Y. Onda, U.S. Patent, 7, 357, 339 (2008).

15. T. Kondo, R. Kose, H. Naito and W. Kasai, Carbohydr. Polym., 112, 284 (2014).

16. T. Kondo, D. Kumon, A. Mieno, Y. Tsujita and R. Kose, Mater. Res. Express, 1, 16 (2014).

17. Y. Tsujita and T. Kondo, J. Fiber Sci. Technol., 75 , 112 (2019).

18. T. Tsuji, K. Tsuboi, S. Yokota, S. Tagawa and T. Kondo, Biomacromolecules, 22, 620 (2021).

19. S. Yokota, K. Kamada, A. Sugiyama and T. Kondo, Carbohydr. Polym., 226. 115293 (2019).

20. K. Ishida, S. Yokota and T. Kondo, Carbohydr. Polym., 261, 117845 (2021).

21. W. Ramsden, Proceedings of the Royal Society of London, 72, 156 (1903).

22. S. U. Pickering, J. Chem. Soc., 91, 2001 (1907).

23. S. Abend, N. Bonnke, U. Gutschner and G. Lagaly, Colloid. Polym. Sci., 276, 730 (1998).

24. B. P. Binks and S. O. Lumsdon, Langmuir, 17, 4540 (2001).

25. L. S. Dorobantu, A. K. C. Yeung, J. M. Foght and M. R. Gray, Appl. Environ., Microbiol., 70, 6333 (2004).

26. H. Ougiya, K. Watanabe, Y. Morinaga and F. Yoshinaga, Biosci. Biotechnol., Biochem., 61, 1541 (1997).

27. E. Dickinson, Food Hydrocolloids, 68, 219 (2017).

28. A. Niroula, T. D. Gamot, C. W. Ooi and S. Dhital, Food Hydrocolloids, 112, 106303 (2021).

29. M. V. Tzoumaki, T. Moschakis, V. Kiosseoglou and C. G. Biliaderis, Food Hydrocolloids, 25. 1521 (2011).

30. Y. Huang, H. Liu, S. Liu and S. Li, J. Agric. Food. Chem., 68, 14620 (2020).

31. Y. Kaku, S. Fujisawa, T. Saito and A. Isogai, Biomacromolecules, 21, 1886 (2020).

32. S. Noguchi, K. Sato, K. Yamamoto and J. Kadokawa, Int. J. Biol. Macromol., 126, 187 (2019).

33. R. Kose, I. Mitani, W. Kasai and T. Kondo, Biomacromolecules, 12, 716 (2011).

34. A. C. Neville, D. A. D. Parry and J. Woodheadgalloway, J. Cell Sci., 21, 73 (1976).

35. G. Ishikawa and T. Kondo, Cellulose, 24, 5495 
(2017).

36. Y. M. Fan, T. Saito and A. Isogai, Carbohydr. Polym., 79, 1046 (2010).

37. M. V. Smoluchowski, Z. Phys. Che., 92, 129 (1918).

38. S. Tagawa, K. Ishida, T. Tsuji and T. Kondo, Cellulose, 28, 2917 (2021).

39. Y. Jia, X. Zhai, W. Fu, Y. Liu, F. Fei and C. Zhong, Carbohydr. Polym., 151, 907 (2016).

40. J. Wu, M. Shi, W. Li, L. Zhao, Z. Wang, X, Yan, W, Norde and Y. Li, Colloids and Surf., 127, 96 (2015).
41. Y. Goi, S. Fujisawa, T. Saito, K. Yamane, K. Kuroda and A. Isogai, Langmuir, 35, 10920 (2019).

42. R. Tanaka, T. Saito, D. Ishii and A. Isogai, Cellulose, 21, 1581 (2014).

43. K. Kamogawa, G. Okudaira, M. Matsumoto, T. Sakai, H. Sakai and M. Abe, Langmuir, 20, 2043 (2004).

44. A. G. S. Prado, J. L. Macedo, S. C. L. Dias and J. A. Dias, Colloids and Surf., B, 35, 23 (2004).

45. C. V. Nikiforidis and E. Scholten, $R S C A d v$., 5, 37789 (2015). 